\title{
Different dietary omega-3 sources during pregnancy and DHA in the developing rat brain
}

\author{
Caroline E. CHILDS ${ }^{1,2}$ \\ Alison L. FEAR ${ }^{1}$ \\ Samuel P. HOILE ${ }^{1}$ \\ Philip C. CALDER ${ }^{1}$ \\ ${ }^{1}$ Institute of Human Nutrition and \\ Developmental Origins of Health and \\ Disease Division, School of Medicine, \\ University of Southampton, \\ Southampton SO16 6YD, United \\ Kingdom \\ $<$ c.e.childs@reading.ac.uk> \\ ${ }^{2}$ Department of Food and Nutritional \\ Sciences, The University of Reading, \\ Whiteknights PO Box 226, Reading, \\ Berkshire RG6 6AP, UK
}

\begin{abstract}
The essential $n-3$ fatty acid $\alpha$-linolenic acid (ALA) can be converted into eicosapentaenoic acid (EPA), docosapentaenoic acid (DPA) and docosahexaenoic acid (DHA) under the action of desaturase and elongase enzymes. Human studies have demonstrated that females convert a higher proportion of ALA into EPA and DHA than males. We have demonstrated that when fed upon an ALA rich diet, female rats have a significantly higher EPA content of plasma and liver lipids than males. When fetal tissues were collected, it was observed that pups from dams fed the ALA rich diet had a comparable brain DHA status to those from dams fed on a salmon-oil based diet, indicating that conversion of ALA to DHA during pregnancy was efficient, and that DHA accumulated in a tissue-specific manner. Similar efficacy of dietary ALA in women during pregnancy would mean that plant $n-3$ fatty acids would be useful alternatives to preformed EPA and DHA.
\end{abstract}

Key words: omega-3, sex, pregnancy

\section{Synthesis of long chain n-3 PUFA}

Dietary sources of the essential fatty acid $\alpha$-linolenic acid (ALA; 18:3n-3) include green leaves, some seeds, nuts and cooking oils. The principal dietary source of the long-chain (LC) n-3 polyunsaturated fatty acids (PUFA) eicosapentaenoic acid (EPA; 20:5n-3), docosapentaenoic acid (DPA; 22:5n-3) and docosahexaenoic acid (DHA; 22:6n-3) is oily fish, yet it is estimated that only $27 \%$ of UK adults habitually eat oily fish (Scientific Advisory Committee on Nutrition, 2004).

In addition to consumption in the diet, LC n-3 PUFA can be endogenously synthesised via a series of elongase, desaturase and $\beta$-oxidation steps from their essential fatty acid precursor ALA (Leonard et al., 2004) (figure 1). This same series of desaturase and elongase enzymes is also involved in the metabolism of the n-6 PUFA linoleic acid (LA) into its longer-chain, more unsaturated derivatives (e.g. arachidonic acid). In Western diets, consumption of LA is about 10 times that of ALA (Burdge and Calder, 2006), suggesting that synthesis of n-6 PUFA will predominate.

\section{Sex and plasma and tissue n-3 fatty acid composition}

Studies have identified sex differences in circulating plasma concentrations of LC n-3 PUFA. While these studies vary in their sample size, degree of dietary control exerted and the range of blood lipids analysed, all have found that women have significantly higher circu- lating DHA concentrations compared to men and that this is independent of dietary intake (Nikkari et al., 1995; Giltay et al., 2004; Bakewell et al., 2006; Crowe et al., 2008). Rat studies have also identified that the proportion of

\begin{tabular}{|ll|}
\hline \multicolumn{2}{|c|}{ Abbreviations } \\
ALA & $\alpha$-linolenic acid \\
DHA & docosahexaenoic acid \\
DPA & docosapentaenoic acid \\
EPA & eicosapentaenoic acid \\
HF & high-fat \\
LA & linoleic acid \\
LC & long-chain \\
LF & low-fat \\
PC & phosphatidylcholine \\
PE & phosphatidylethanolamine \\
PUFA & polyunsaturated fatty acid \\
\hline
\end{tabular}

To cite this article: Childs CE, Fear AL, Hoile SP, Calder PC. Different dietary omega-3 sources during pregnancy and DHA in the developing rat brain. OCL 2011;18(5):259-262. doi : 10.1684/ocl.2011.0391 


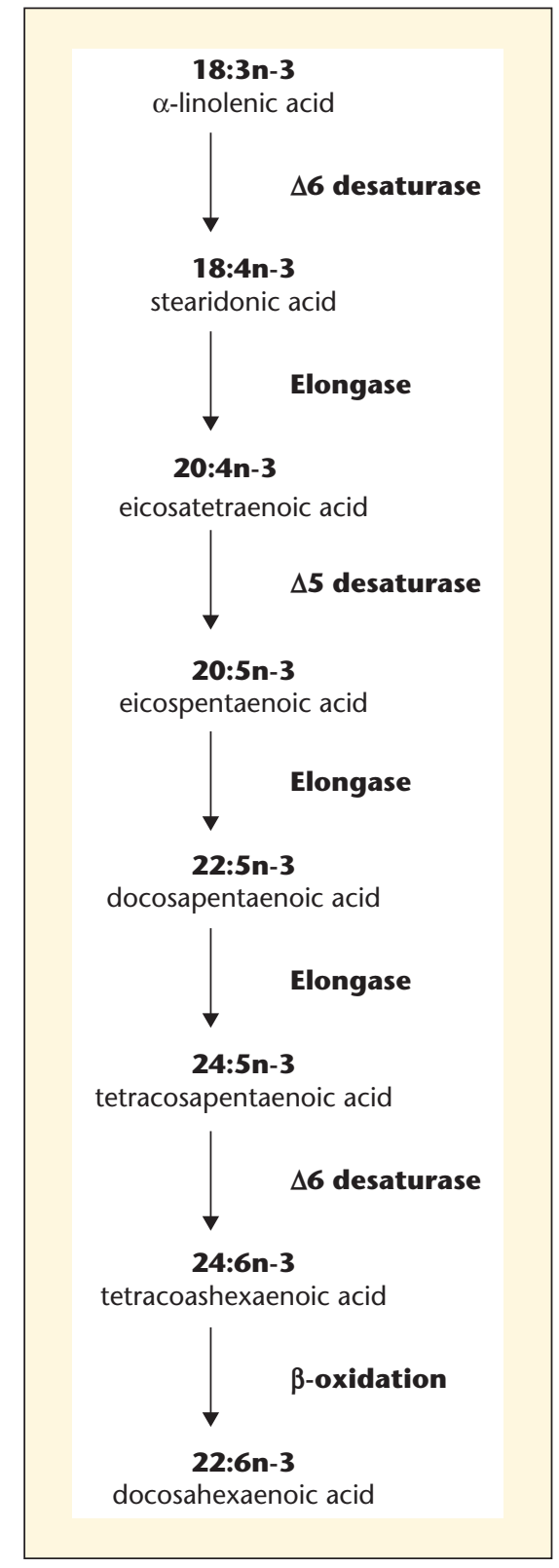

Figure 1. Biosynthesis of LC $n-3$ PUFA from $\alpha$ linolenic acid.

DHA is higher in liver and plasma phospholipids in females than males (Burdge et al., 2008; Extier et al., 2010; Childs et al., 2010a).

Data from studies using stable isotopelabelled ALA demonstrate that there are sex differences in the ability to synthesize LC n-3 PUFA from ALA. Young women converted a greater proportion of ALA into EPA and DHA compared to men (Burdge et al., 2002a; Burdge and Wootton, 2002b). It has been hypoth- esised that sex differences are established in order to ensure an adequate supply of LC n-3 PUFA to the developing fetus during pregnancy (Bakewell, 2006). If this is the case, then it is possible that LC n-3 PUFA synthesis may be further upregulated during pregnancy.

\section{N-3 fatty acids and pregnancy}

Specific maternal dietary fatty acids, particularly n-3 PUFA, have been demonstrated to be essential for successful fetal development and later tissue function in both humans and animals. Transfer of DHA to the developing fetus in human pregnancy predominantly occurs in the last 10 weeks of pregnancy, with the majority of this DHA accumulated within fetal adipose tissue (Haggarty, 2004). The observation that DHA is found in high concentrations in the retina and accumulates in the fetal brain during late pregnancy and in early neonatal life has led to the suggestion that an adequate dietary supply of this fatty acid is required for optimal brain and visual development (Farquharson et al., 1995). Animal studies where n-3 fatty acid deficient diets have been provided demonstrate that dietary $n-3$ fatty acids are essential for normal cognitive and visual function, as reviewed in detail elsewhere (Lauritzen et al., 2001).

Human studies have investigated the role of LC n-3 PUFA, particularly DHA, when provided in milk formula to both preterm and healthy term infants. Metaanalyses indicate that the addition of DHA to pre-term infant formula is beneficial for optimal visual development in early life (Sangiovanni et al., 2000; Uauy et al., 2003). Whether these effects persist beyond early life (i.e. after 4 months of age) has not yet been established. In term infants, formula containing DHA was found to improve markers of cognitive function (Cheatham et al., 2006). However, the clinical relevance of the reported statistically significant differences and the validity of the neurodevelopmental tests utilised in these studies have been questioned (Koo, 2003).

Human studies have demonstrated that there are significant effects of pregnancy upon blood lipid fatty acid composition, though the effects observed have been mixed. For example, while some studies

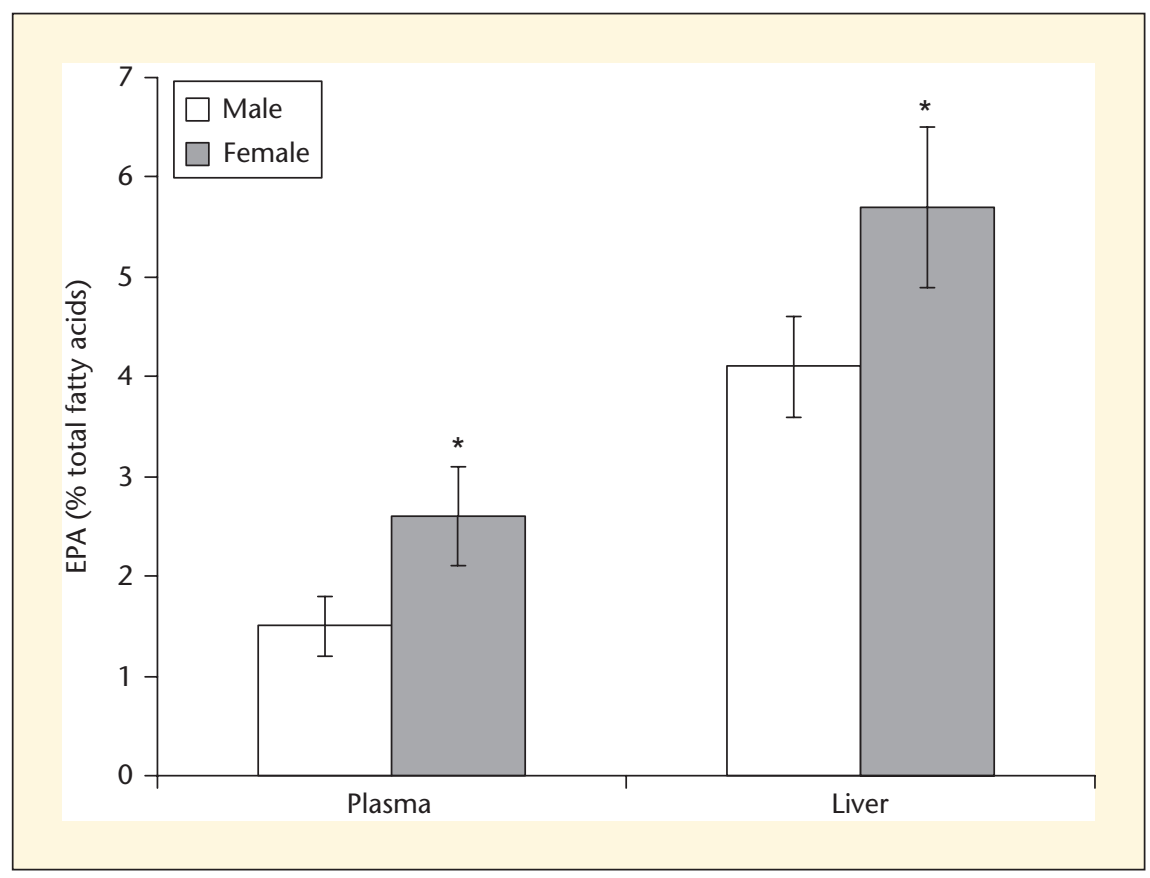

Figure 2. EPA content of plasma phosphatidylcholine in male and female rats fed an ALA-rich diet for 20 days. Values are mean $\pm S D, n=6$ per group. *Significantly different from males $(p<0.05)$. Data are taken from [10]. 
have identified a reduction in plasma phospholipid DHA status during pregnancy (Wijendran et al., 1999; Hornstra, 2000), others have reported increased DHA content of plasma phospholipids (Postle et al., 1995; Burdge et al., 2006) or red blood cells (Stewart et al., 2007). These differences between studies can most likely be attributed to variations in the type of sample analysed, whether results were expressed as a percentage or in absolute concentrations, and the possible confounding effect of maternal diet and adipose tissue composition. Studies in rats comparing virgin animals with those at the end of pregnancy have shown that the fatty acid composition of phospholipids from plasma and liver is significantly altered in response to pregnancy with higher DHA and lower arachidonic acid contents (Smith and Walsh, 1975; Cunnane, 1989; Chen et al., 1992; Burdge et al., 1994). In rats, higher DHA in liver and plasma phosphatidylcholine (PC) has been attributed to changes in PC synthesis (Burdge et al., 1994). However, it is unclear whether the increased availability of DHA is a result of mobilisation of DHA from adipose tissue, increased dietary intake or greater synthesis by desaturation and elongation of precursors.

\section{Use of an ALA rich diet in rat models}

We have identified that there are significant diet $\times$ sex interactions in rat tissue $\mathrm{n}-3$ fatty acid composition (Childs et al., 2010a). Female rats fed an ALA rich diet had a higher proportion of EPA in plasma and liver PC compared to males (figure 2), with data suggesting that these differences may be mediated by higher expression of $\Delta 6$ desaturase $(\Delta 6 \mathrm{D}) \mathrm{mRNA}$ and greater $\triangle 6 \mathrm{D}$ activity in females than males (Childs et al., 2010a). We also identified that providing an ALA rich diet during pregnancy resulted in equivalent EPA status in fetal immune tissues (figure $3 A$ ) and equivalent DHA status in the fetal brain to that achieved in the offspring of dams fed a highfat salmon-oil diet (figure 3B) (Childs et al., 2010b). This indicates a significant role of maternal and/or fetal LC n-3 PUFA synthesis in determining fetal LC $\mathrm{n}-3$ PUFA status in a tissue specific manner. The effect of maternal diet during pregnancy upon fetal brain DHA content persists until weaning (figure 4).

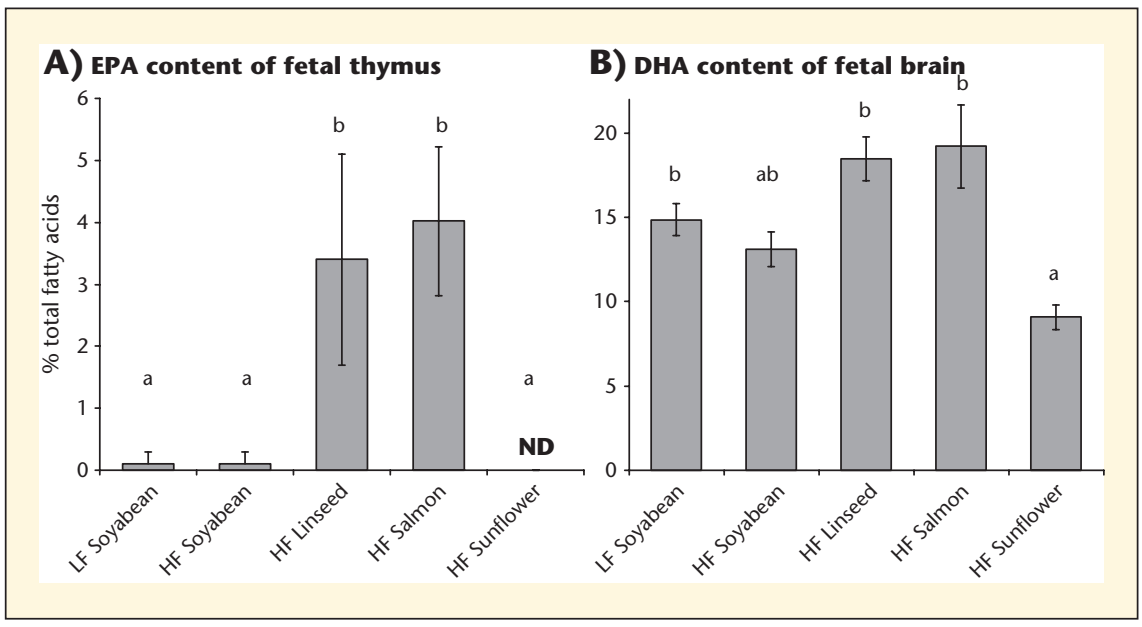

Figure 3. EPA and DHA contents of thymus and brain phosphatidylethanolamine from 20-day gestation pups from rat dams fed different experimental diets for 20 days of pregnancy. Values are mean $\pm S D, n=6$ per group. Means without a common letter differ, $P<0.05$. $L F$, low fat diet; $H F$, high fat diet; ND, not detected $(<0.1 \%)$. Data are taken from [29].

\section{Discussion}

We have found that the percentage content of $\mathrm{n}-3$ fatty acids among rats receiving standard laboratory chow ad libitum and the response of rats to ALA supplementation regimes compares favourably with available data from human studies. If dietary ALA during pregnancy significantly influen-

ces fetal brain and immune tissue LC $\mathrm{n}-3$ PUFA content in humans this would have significant implications for strategies aimed at improving infant cognitive function or promoting infant immune development and reducing the risk of immune dysfunction (e.g. atopic sensitisation). To date, studies in pregnancy examining these infant

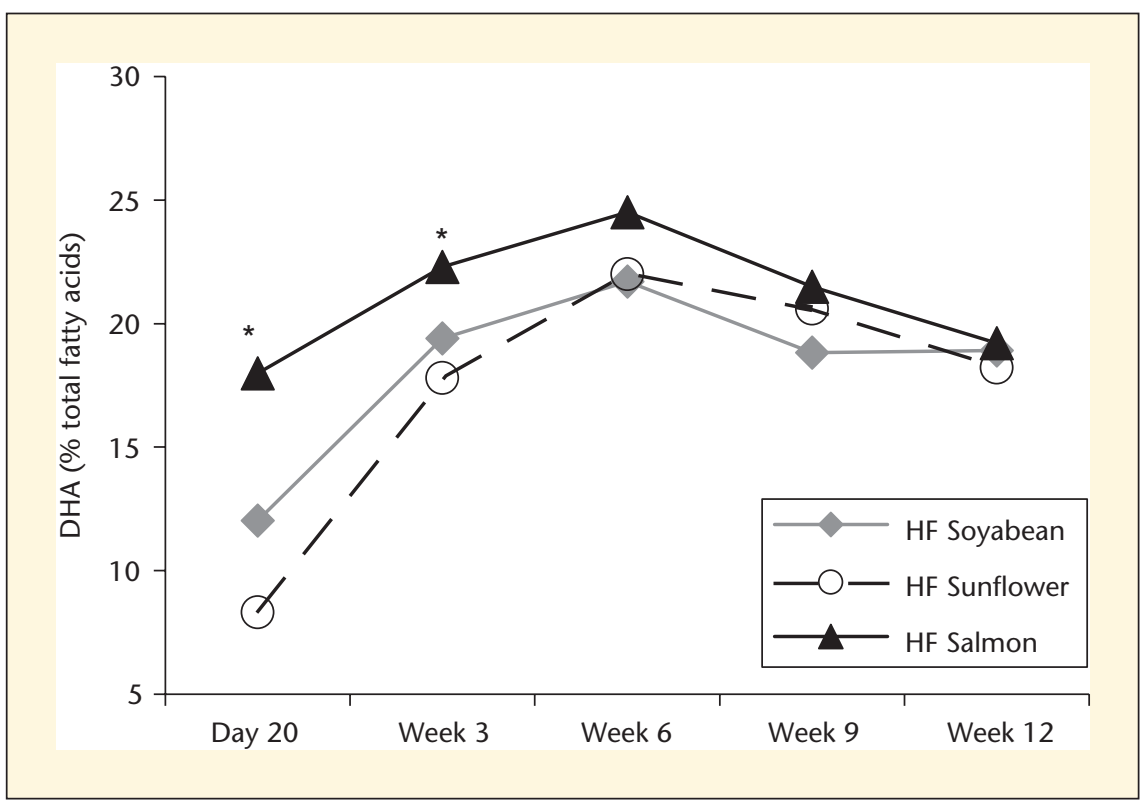

Figure 4. DHA content of brain phosphatidylethanolamine of pups from rat dams fed different high fat (HF) experimental diets for 20 days of pregnancy. Day 20 indicates day 20 of gestation; week 3, 6, 9 and 12 indicate weeks post-birth. Values are mean $n=6$ per group. ${ }^{*} H F$ Salmon significantly different from the other two groups, $P<0.05$. Data are not previously published. 
outcomes have largely provided marinesources of $n-3$ fatty acids. The availability of plant-oil sources of n-3 fatty acids would greatly benefit vegetarian and vegan women and would have an environmental impact by reducing demand upon marine resources.

Further rat studies will be necessary to determine the threshold of ALA supplementation required to maintain equivalent brain DHA and immune tissue EPA to that achieved with a fish-oil rich diet. Whether these changes to tissue fatty acid composition result in any differences in offspring visual, cognitive or immune function is also yet to be determined. It would be of interest to conduct human studies to investigate whether there are similar sex differences in the response to dietary ALA. If the effects observed in our rat model of dietary ALA during pregnancy were replicated in human studies, this approach could be used to investigate whether there are benefits to offspring health, including women who are unwilling or unable to consume marine-based interventions (i.e. fish or fish oils).

\section{REFERENCES}

Bakewell L, Burdge GC, Calder PC. Polyunsaturated fatty acid concentrations in young men and women consuming their habitual diets. Br J Nutr 2006; 96: 93-9.

Burdge GC, Hunt AN, Postle AD. Mechanisms of hepatic phosphatidylcholine synthesis in adult rat: effects of pregnancy. Biochem J 1994; 303: 941-7.

Burdge GC, Jones AE, Wootton SA. Eicosapentaenoic and docosapentaenoic acids are the principal products of alpha-linolenic acid metabolism in young men. Br / Nutr 2002a; 88: 355-63.

Burdge GC, Wootton SA. Conversion of alpha-linolenic acid to eicosapentaenoic, docosapentaenoic and docosahexaenoic acids in young women. Br / Nutr 2002b; 88: 411-20.

Burdge GC, Calder PC. Dietary alpha-linolenic acid and health related outcomes: a metabolic perspective. Nutr Res Rev 2006; 19: 26-52.

Burdge GC, Sherman RC, Ali Z, Wootton SA, Jackson AA. Docosahexaenoic acid is selec- tively enriched in plasma phospholipids during pregnancy in Trinidadian womenresults of a pilot study. Reprod Nutr Dev 2006; 46: 63-7.

Burdge GC, Slater-Jefferies JL, Grant RA, et al. Sex, but not maternal protein or folic acid intake, determines the fatty acid composition of hepatic phospholipids, but not of triacylglycerol, in adult rats. Prostaglandins Leukot Essent Fatty Acids 2008; 78: 73-9.

Cheatham CL, Colombo J, Carlson SE. N-3 fatty acids and cognitive and visual acuity development: methodologic and conceptual considerations. Am / Clin Nutr 2006; 83: 1458S-66S.

Chen ZY, Yang JL, Menard CR, Cunnane SC. Linoleoyl-enriched triacylglycerol species increase in maternal liver during late pregnancy in the rat. Lipids 1992; 27: 21-4.

Childs CE, Romeu-Nadal M, Burdge GC, Calder PC. The polyunsaturated fatty acid composition of hepatic and plasma lipids differ by both sex and dietary fat intake in rats. J Nutr 2010a; 140: 245-50.

Childs CE, Romijn T, Enke U, Hoile S, Calder PC. Maternal diet during pregnancy has tissue-specific effects upon fetal fatty acid composition and alters fetal immune parameters. Prostaglandins Leukot Essent Fatty Acids 2010b; 83: 179-84.

Crowe FL, Skeaff CM, Green TJ. Serum n-3 long-chain PUFA differ by sex and age in a population-based survey of New Zealand adolescents and adults. Br / Nutr 2008; 99: 168-74.

Cunnane SC. Changes in essential fatty acid composition during pregnancy: maternal liver, placenta and fetus. Nutrition 1989; 5: 253-5.

Extier A, Langeliera B, Perruchota $\mathrm{MH}$, et al. Gender affects liver desaturase expression in a rat model of n-3 fatty acid repletion. / Nutr Biochem 2010; 21: 180-7.

Farquharson J, Jamieson EC, Logan RW, Patrick WJ, Howatson AG, Cockburn F. Age- and dietary-related distributions of hepatic arachidonic and docosahexaenoic acid in early infancy. Pediatr Res 1995; 38: 361-5.

Giltay EJ, Gooren LJ, Toorians AW, Katan MB, Zock PL. Docosahexaenoic acid concentrations are higher in women than in men because of estrogenic effects. Am / Clin Nutr 2004; 80: 1167-74.
Haggarty P. Effect of placental function on fatty acid requirements during pregnancy. Eur J Clin Nutr 2004; 58: 1559-70.

Hornstra G. Essential fatty acids in mothers and their neonates. Am / Clin Nutr 2000; 71: 1262S-9S.

Koo WW. Efficacy and safety of docosahexaenoic acid and arachidonic acid addition to infant formulas: can one buy better vision and intelligence? J Am Coll Nutr 2003; 22: 101-7.

Lauritzen L, Hansen HS, Jorgensen $\mathrm{MH}$, Michaelsen KF. The essentiality of long chain $\mathrm{n}-3$ fatty acids in relation to development and function of the brain and retina. Prog Lipid Res 2001; 40: 1-94.

Leonard AE, Pereira SL, Sprecher $H$, Huang YS. Elongation of long-chain fatty acids. Prog Lipid Res 2004; 43: 36-54.

Nikkari T, Luukkainen P, Pietinen P, Puska P. Fatty acid composition of serum lipid fractions in relation to gender and quality of dietary fat. Ann Med 1995; 27: 491-8.

Postle AD, Al MDM, Burdge GC, Hornstra G. The composition of individual molecular species of plasma phosphatidylcholine in human pregnancy. Early Hum Dev 1995; 43: 47-58.

Sangiovanni JP, Parra-Cabrera S, Colditz GA, Berkey CS, Dwyer JT. Meta-analysis of dietary essential fatty acids and long-chain polyunsaturated fatty acids as they relate to visual resolution acuity in healthy preterm infants. Pediatrics 2000; 105: 1292-8.

Scientific Advisory Committee on Nutrition ( $\mathrm{SACON}$ ). Advice on fish consumption: benefits \& risks. London: TSO, 2004.

Smith RW, Walsh A. Composition of liver lipids of the rat during pregnancy and lactation. Lipids 1975; 10: 643-5.

Stewart F, Rodie VA, Ramsay JE, Greer IA, Freeman DJ, Meyer BJ. Longitudinal assessment of erythrocyte fatty acid composition throughout pregnancy and post partum. Lipids 2007; 42: 335-44.

Uauy R, Hoffman DR, Mena P, Llanos A, Birch EE. Term infant studies of DHA and ARA supplementation on neurodevelopment: results of randomized controlled trials. J Pediatr 2003; 143: S17-S25.

Wijendran V, Bendel RB, Couch SC, et al. Maternal plasma phospholipid polyunsaturated fatty acids in pregnancy with and without gestational diabetes mellitus: relations with maternal factors. Am / Clin Nutr 1999; 70: 53-61. 